\title{
A branch-and-price algorithm to solve the integrated berth allocation and yard assignment problem in bulk ports
}

\author{
Tomáš Robenek
}

$1^{\text {st }}$ Workshop on Large Scale Optimization

November 21, 2012 


\section{Agenda}

1 Introduction

2 Problem Definition

3 Branch \& Price

4 Results

5 Conclusion

6 Future Work

TRANSP-OR 
1 Introduction

- Motivation

- References

2 Problem Definition

3 Branch \& Price

年

TRANSP-DR 


\section{Motivation}

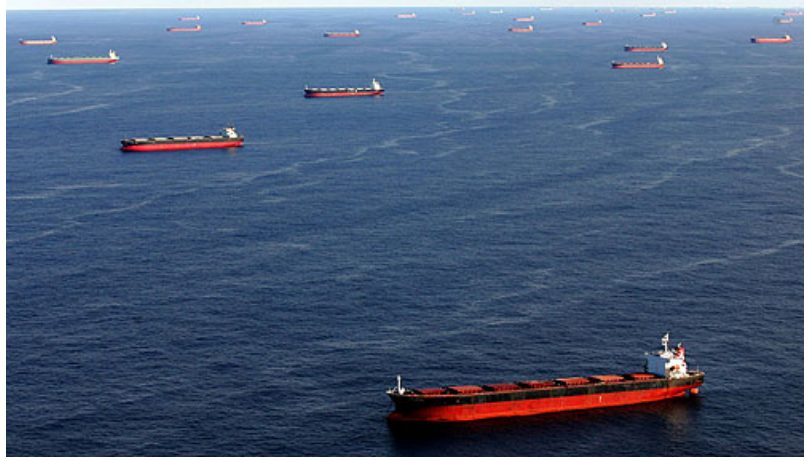

Figure: Vessels queueing at Newcastle port, Australia (queue hits 60 vessels as max.) 


\section{References}

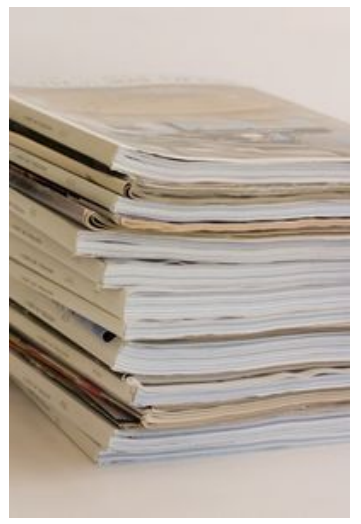

诖 UANG, N., M.Bierlaire, AND VACCA, I.

Exact and heuristic approach methods to solve berth allocation problem in bulk ports.

Tech. rep., TRANSP-OR, Ecole Polytechnique Federale De Lausanne, 2012.

Z ZHEN, L.

An integrated model for berth template and yard template planning in transshipment hubs.

Transportation Science 45 (2011), 483-504. 


\section{Problem Definition}

- Berth Allocation Problem

- Yard Assignment Problem

- Objective 


\section{Berth Allocation Problem}

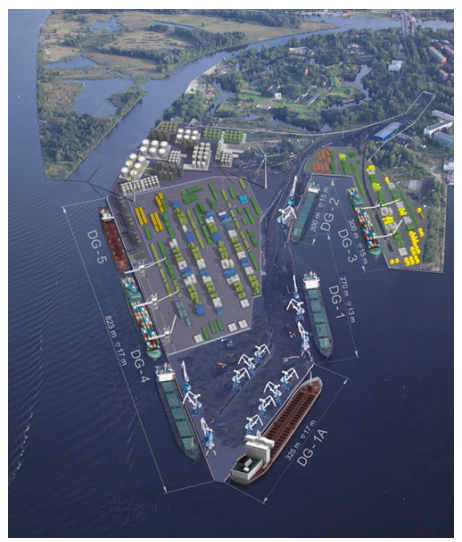

Figure: Lacon Itd.'s plan for extension of the Riga's port, Latvia 


\section{Yard Assignment Problem}

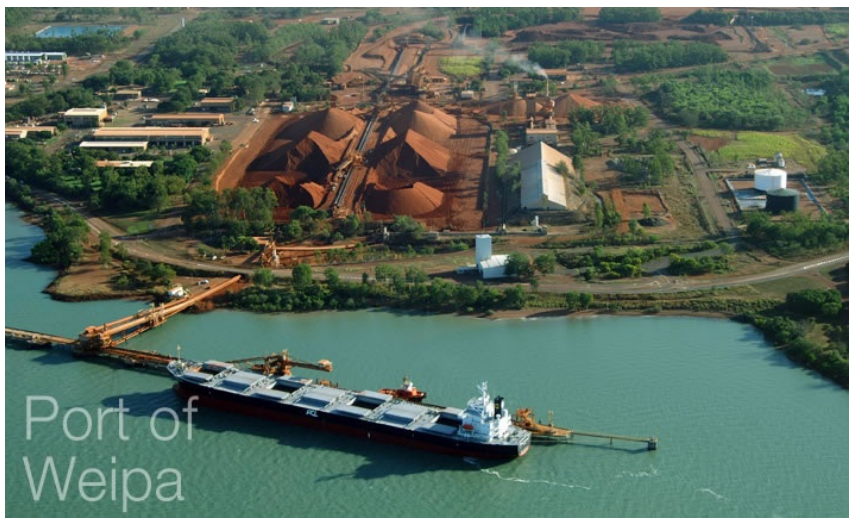

Figure: Port of Weipa, Queensland, Australia 


\section{Objective coto model}

- Minimize Handling Time + Delay = Service Time

- Obtain berth schedule

- Obtain plan of a yard storage

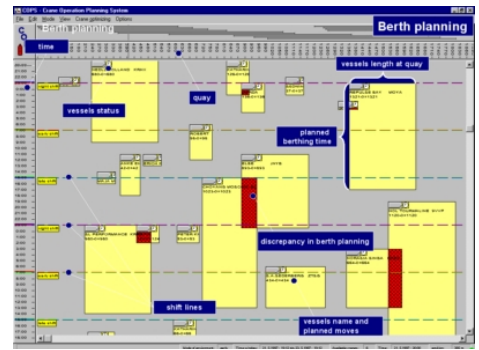


3 Branch \& Price

- Framework

- Initial Solution

- Master Problem

- Sub-Problem

- Branch and Bound

- Improvement Methods 


\section{Framework}

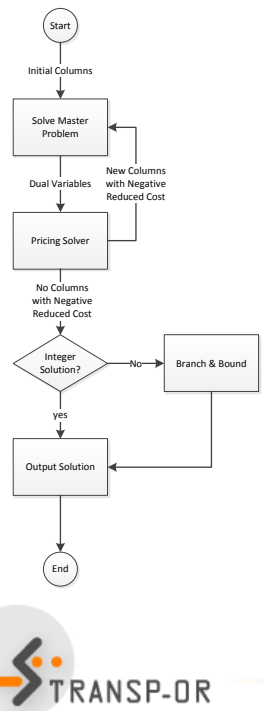

- Initial Solution

- Column Generation - Lower Bound

- Branch and Bound - Optimal Integer Solution 


\section{Initial Solution}
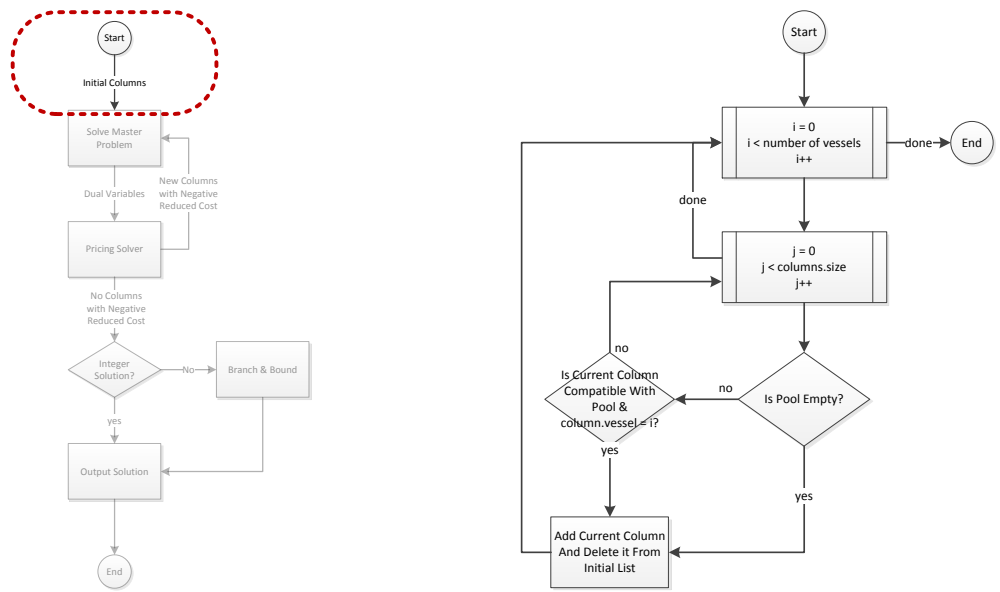

TRANSP-DR 


\section{Master Problem}

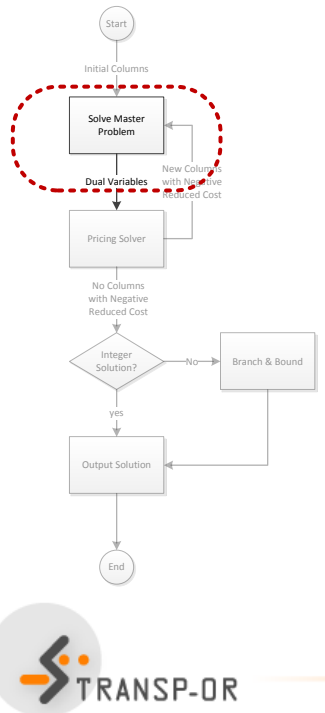

Idea

- SP Go to SP

- relaxation of $\lambda$ and $\mu$

- $\Omega$ reduced to $\Omega_{1}$ 


\section{Sub-Problem crotomoded}

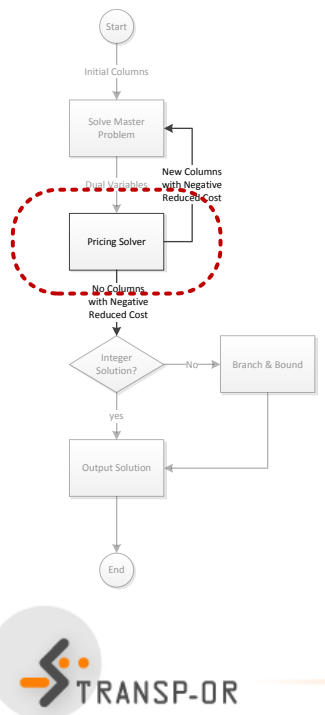

\section{Idea}

- run for each vessel separately

" get $\leq 40 \cdot n$ columns

Dual Variables

" $\alpha, \beta_{k t}, \gamma_{l t}, \delta_{l w}$ 


\section{Branch and Bound}
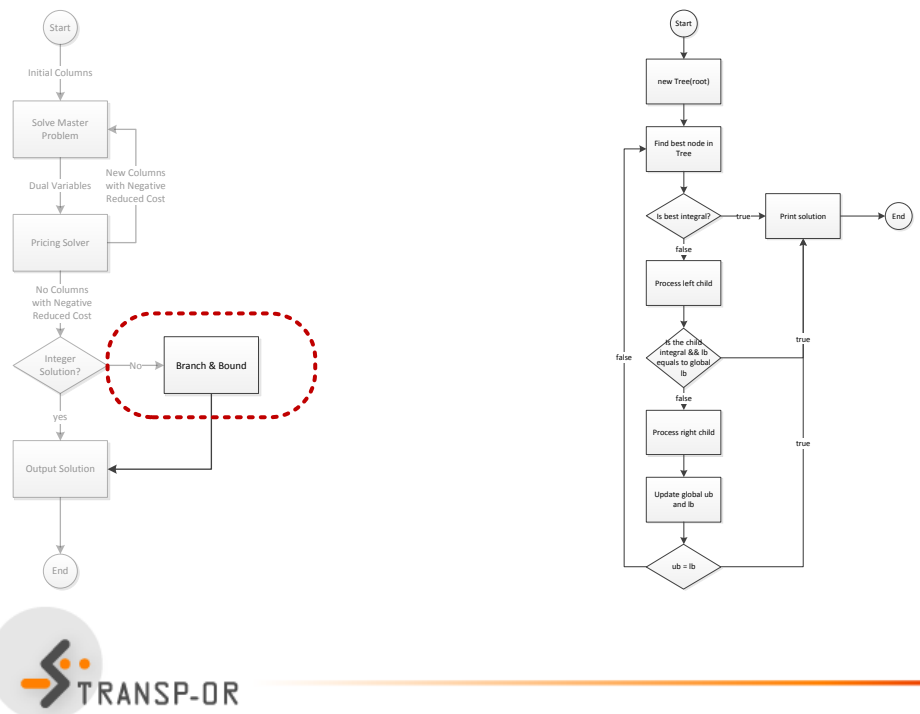


\section{Improvement Methods - general + stabilization}

\section{General}

- decomposition

- 40 columns/sub-problem/iteration

$$
\begin{gathered}
(c+s-a)-\left(\alpha+\sum_{k \in K} \sum_{t \in T} \beta_{k t} \cdot \text { beta }_{k t}+\right. \\
\left.\sum_{l \in L} \sum_{t \in T} \gamma_{l t} \cdot \text { gamma }_{l t}+\sum_{l \in L} \sum_{w \in W} \delta_{l w} \cdot \text { delta }_{\mid w}\right) \leq 0
\end{gathered}
$$

\section{Stabilization of duals}

- assumption: duals oscilate

- solution: $\overline{\alpha_{i}}=\epsilon \cdot \overline{\alpha_{i}}+(1-\epsilon) \cdot \alpha_{i}$ 


\section{Improvement Methods - aggregation 1/2}

\begin{tabular}{c|cc|ccc} 
row & section & time & column 1 & column 2 & column 3 \\
\hline 1 & 1 & 1 & 1 & 0 & 0 \\
2 & 1 & 2 & 1 & 0 & 0 \\
3 & 1 & 3 & 1 & 0 & 0 \\
4 & 1 & 4 & 0 & 0 & 0 \\
5 & 1 & 5 & 0 & 0 & 0 \\
6 & 1 & 6 & 0 & 0 & 0 \\
\hline 7 & 2 & 1 & 0 & 0 & 0 \\
8 & 2 & 2 & 0 & 0 & 0 \\
9 & 2 & 3 & 0 & 1 & 0 \\
10 & 2 & 4 & 0 & 1 & 0 \\
11 & 2 & 5 & 0 & 1 & 0 \\
12 & 2 & 6 & 0 & 1 & 0 \\
\hline 13 & 3 & 1 & 0 & 0 & 0 \\
14 & 3 & 2 & 0 & 0 & 1 \\
15 & 3 & 3 & 0 & 1 & 1 \\
16 & 3 & 4 & 0 & 1 & 1 \\
17 & 3 & 5 & 0 & 1 & 0 \\
18 & 3 & 6 & 0 & 1 & 0
\end{tabular}




\section{Improvement Methods - aggregation 2/2}

\begin{tabular}{c|cc|ccc} 
row & section & time & column 1 & column 2 & column 3 \\
\hline 1 & 1 & 1 & 1 & 0 & 0 \\
2 & 1 & 4 & 0 & 0 & 0 \\
3 & 2 & 3 & 0 & 1 & 0 \\
4 & 3 & 2 & 0 & 0 & 1 \\
5 & 3 & 3 & 0 & 1 & 1
\end{tabular}


4 Results

- Set Partitioning

- Column Generation

- Stabilized Method

- Aggregated Method

- Branch and Bound 


\section{Set Partitioning}

\begin{tabular}{|c|c|c|c|c|c|}
\hline \multicolumn{3}{|c|}{ Instance } & \multicolumn{2}{|c|}{ RMP } & \multirow[b]{2}{*}{$\Omega$} \\
\hline \# vessels & \# sections & congestion & time & value & \\
\hline \multirow{4}{*}{10} & \multirow{2}{*}{10} & no & $1 \mathrm{~m} \mathrm{03s}$ & 202 & 121863 \\
\hline & & yes & $3 \mathrm{~m} \mathrm{35s}$ & 219 & 149163 \\
\hline & \multirow{2}{*}{20} & no & $9 \mathrm{~m} \mathrm{35s}$ & 306 & 213268 \\
\hline & & yes & $24 \mathrm{~m} \mathrm{08s}$ & 316 & 265138 \\
\hline \multirow{4}{*}{15} & \multirow{2}{*}{10} & no & $2 \mathrm{~m} \mathrm{27s}$ & 301 & 162846 \\
\hline & & yes & $3 \mathrm{~m} \mathrm{52s}$ & 328 & 210246 \\
\hline & \multirow{2}{*}{20} & no & $27 \mathrm{~m} \mathrm{13s}$ & 450 & 284086 \\
\hline & & yes & $1 \mathrm{~h} 21 \mathrm{~m} 15 \mathrm{~s}$ & 468 & 374146 \\
\hline \multirow{4}{*}{20} & \multirow{2}{*}{10} & no & $3 \mathrm{~m} \mathrm{36s}$ & 384 & 181373 \\
\hline & & yes & $11 \mathrm{~m} \mathrm{36s}$ & 421 & 263173 \\
\hline & \multirow{2}{*}{20} & no & $40 m$ 09s & 567 & 312515 \\
\hline & & yes & - & - & 467935 \\
\hline
\end{tabular}




\section{Column Generation}

\begin{tabular}{|c|c|c|c|c|c|c|c|c|c|c|}
\hline \multicolumn{3}{|c|}{ Instance } & \multicolumn{2}{|c|}{ Column Generation } & \multirow[b]{2}{*}{$\Omega_{1}$} & \multirow[b]{2}{*}{$\mathrm{lb}$} & \multirow[b]{2}{*}{$u b$} & \multirow[b]{2}{*}{ gap1 } & \multirow[b]{2}{*}{ gap2 } & \multirow[b]{2}{*}{ gap } \\
\hline \# vessels & \# sections & congestion & time & \# iter. & & & & & & \\
\hline \multirow{4}{*}{10} & \multirow{2}{*}{10} & no & $2 \mathrm{~m} \mathrm{07s}$ & 4 & 587 & 197 & 206 & $2 \%$ & $2 \%$ & $4 \%$ \\
\hline & & yes & $6 \mathrm{~m} \mathrm{28s}$ & 7 & 1300 & 213 & 221 & $3 \%$ & $1 \%$ & $4 \%$ \\
\hline & \multirow{2}{*}{20} & no & $4 m 49 s$ & 4 & 696 & 306 & 309 & $0 \%$ & $1 \%$ & $1 \%$ \\
\hline & & yes & $18 \mathrm{~m} 50 \mathrm{~s}$ & 8 & 1454 & 311 & 316 & $2 \%$ & $0 \%$ & $2 \%$ \\
\hline \multirow{4}{*}{15} & \multirow{2}{*}{10} & no & $3 \mathrm{~m} \mathrm{25s}$ & 4 & 922 & 293 & 306 & $3 \%$ & $2 \%$ & $4 \%$ \\
\hline & & yes & $26 \mathrm{~m} \mathrm{24s}$ & 7 & 2123 & 314 & 328 & $4 \%$ & $0 \%$ & $4 \%$ \\
\hline & \multirow{2}{*}{20} & no & $13 \mathrm{~m} \mathrm{22s}$ & 5 & 1308 & 447 & 452 & $1 \%$ & $0 \%$ & $1 \%$ \\
\hline & & yes & $1 \mathrm{~h} 7 \mathrm{~m} 4 \mathrm{~s}$ & 12 & 3270 & 457 & 471 & $2 \%$ & $1 \%$ & $3 \%$ \\
\hline \multirow{4}{*}{20} & \multirow{2}{*}{10} & no & $3 m 43 s$ & 4 & 1099 & 372 & 390 & $3 \%$ & $2 \%$ & $5 \%$ \\
\hline & & yes & $38 \mathrm{~m} \mathrm{59s}$ & 9 & 3077 & 396 & 435 & $6 \%$ & $3 \%$ & $9 \%$ \\
\hline & \multirow{2}{*}{20} & no & $16 \mathrm{~m} 05 \mathrm{~s}$ & 5 & 1780 & 562 & 572 & $1 \%$ & $1 \%$ & $2 \%$ \\
\hline & & yes & $3 \mathrm{~h} 27 \mathrm{~m} 45 \mathrm{~s}$ & 18 & 4736 & 583 & 625 & - & - & $7 \%$ \\
\hline
\end{tabular}




\section{Column Generation}

\begin{tabular}{|c|c|c|c|c|c|c|c|c|c|c|}
\hline \multicolumn{3}{|c|}{ Instance } & \multicolumn{2}{|c|}{ Column Generation } & \multirow[b]{2}{*}{$\Omega_{1}$} & \multirow[b]{2}{*}{$\mathrm{lb}$} & \multirow[b]{2}{*}{$u b$} & \multirow[b]{2}{*}{ gap1 } & \multirow[b]{2}{*}{ gap2 } & \multirow[b]{2}{*}{ gap } \\
\hline \# vessels & \# sections & congestion & time & \# iter. & & & & & & \\
\hline & & & $2 \mathrm{~m} \mathrm{07s}$ & 4 & 587 & 197 & 206 & $2 \%$ & $2 \%$ & $4 \%$ \\
\hline & & & $6 \mathrm{~m} \mathrm{28s}$ & 7 & 1300 & 213 & 221 & $3 \%$ & $1 \%$ & $4 \%$ \\
\hline & & & $4 m 49 s$ & 4 & 696 & 306 & 309 & $0 \%$ & $1 \%$ & $1 \%$ \\
\hline & & yes & $18 \mathrm{~m} 50 \mathrm{~s}$ & 8 & 1454 & 311 & 316 & $2 \%$ & $0 \%$ & $2 \%$ \\
\hline \multirow{4}{*}{15} & \multirow{2}{*}{10} & no & $3 \mathrm{~m} \mathrm{25s}$ & 4 & 922 & 293 & 306 & $3 \%$ & $2 \%$ & $4 \%$ \\
\hline & & & $26 \mathrm{~m} \mathrm{24s}$ & 7 & 2123 & 314 & 328 & $4 \%$ & $0 \%$ & $4 \%$ \\
\hline & \multirow{2}{*}{20} & no & $13 \mathrm{~m} \mathrm{22s}$ & 5 & 1308 & 447 & 452 & $1 \%$ & $0 \%$ & $1 \%$ \\
\hline & & yes & $1 \mathrm{~h} 7 \mathrm{~m} 4 \mathrm{~s}$ & 12 & 3270 & 457 & 471 & $2 \%$ & $1 \%$ & $3 \%$ \\
\hline \multirow{4}{*}{20} & \multirow{2}{*}{10} & no & $3 \mathrm{~m} \mathrm{43s}$ & 4 & 1099 & 372 & 390 & $3 \%$ & $2 \%$ & $5 \%$ \\
\hline & & & $38 \mathrm{~m} \mathrm{59s}$ & 9 & 3077 & 396 & 435 & $6 \%$ & $3 \%$ & $9 \%$ \\
\hline & \multirow{2}{*}{20} & no & $16 \mathrm{~m} \mathrm{05s}$ & 5 & 1780 & 562 & 572 & $1 \%$ & $1 \%$ & $2 \%$ \\
\hline & & yes & $3 \mathrm{~h} 27 \mathrm{~m} 45 \mathrm{~s}$ & 18 & 4736 & 583 & 625 & - & - & $7 \%$ \\
\hline
\end{tabular}




\section{Column Generation - Duals}

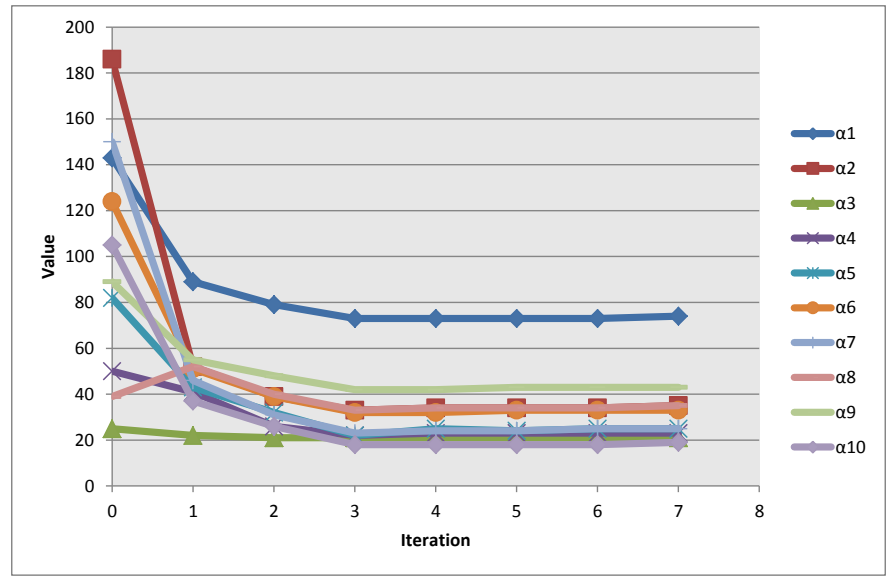

TRANSP-DR

\section{(APFl}

ECOLE POLYTICHNIQUE FEDTRLI DE LAUSANNE

$22 / 35$ 


\section{Column Generation - Time Consumption}

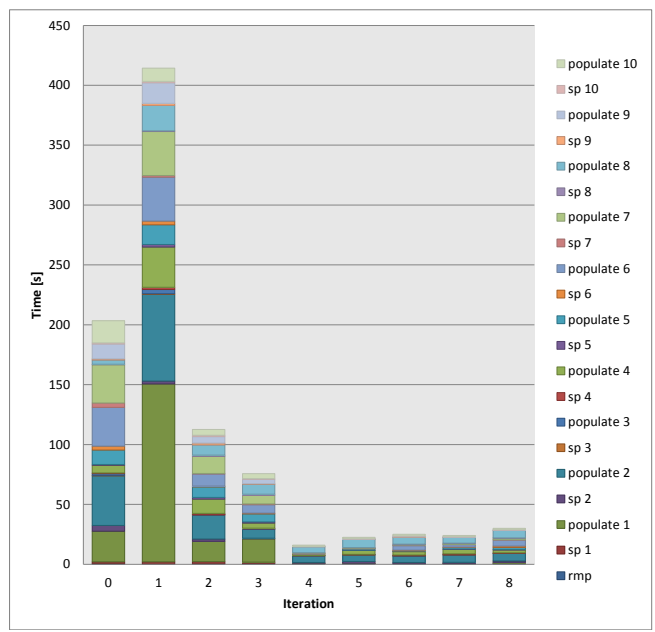

TRANSP-OR 


\section{Column Generation - Pool}

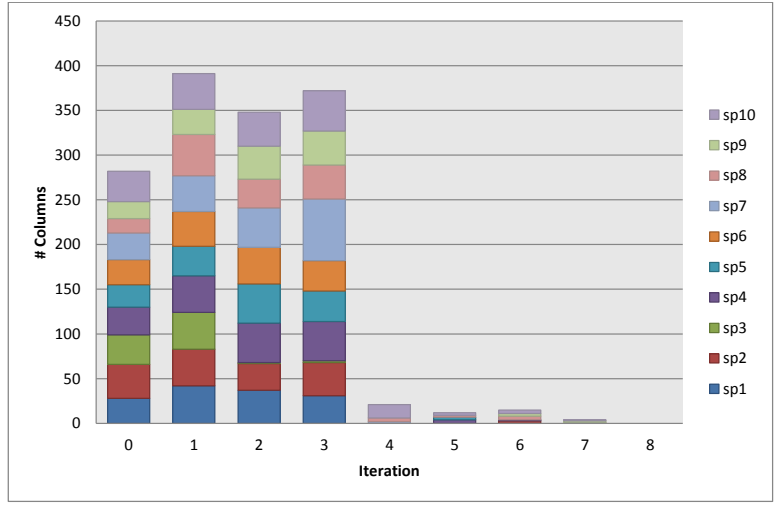

TRANSP-DR 


\section{Column Generation - Objective Fce Evolution}

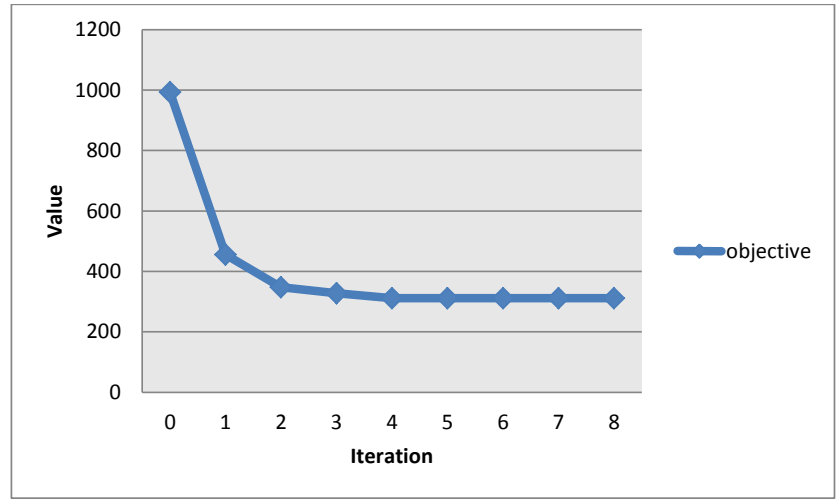

TRANSP-OR 


\section{Stabilized Method}

\begin{tabular}{|c|c|c|c|c|c|c|c|}
\hline \multirow[b]{2}{*}{$\epsilon$} & \multirow[b]{2}{*}{$\Delta$} & \multicolumn{3}{|c|}{ Uncongested } & \multicolumn{3}{|c|}{ Congested } \\
\hline & & iter. & time & pool size & iter. & time & pool size \\
\hline \multirow{3}{*}{1.00} & 0.05 & 15 & $19 \mathrm{~m} \mathrm{15s}$ & 357 & 35 & $1 \mathrm{~h} 7 \mathrm{~m} \mathrm{12s}$ & 560 \\
\hline & 0.10 & 14 & $12 \mathrm{~m} \mathrm{31s}$ & 390 & 37 & $50 \mathrm{~m} \mathrm{17s}$ & 565 \\
\hline & 0.20 & 11 & $10 \mathrm{~m} \mathrm{59s}$ & 397 & 26 & $44 \mathrm{~m} \mathrm{53s}$ & 624 \\
\hline \multirow{3}{*}{0.75} & 0.05 & 10 & $8 \mathrm{~m} \mathrm{43s}$ & 403 & 21 & $45 \mathrm{~m} \mathrm{41s}$ & 642 \\
\hline & 0.10 & 12 & $8 m 38 s$ & 400 & 21 & $40 \mathrm{~m} \mathrm{11s}$ & 642 \\
\hline & 0.20 & 9 & $7 \mathrm{~m} \mathrm{37s}$ & 407 & 21 & $39 m$ 8s & 642 \\
\hline \multirow{3}{*}{0.50} & 0.05 & 7 & $4 \mathrm{~m} \mathrm{27s}$ & 397 & 11 & $23 \mathrm{~m} \mathrm{15s}$ & 724 \\
\hline & 0.10 & 7 & $3 m 58 s$ & 397 & 11 & $21 \mathrm{~m} \mathrm{2s}$ & 724 \\
\hline & 0.20 & 7 & $3 \mathrm{~m} \mathrm{51s}$ & 397 & 11 & $19 \mathrm{~m} \mathrm{29s}$ & 724 \\
\hline \multirow{3}{*}{0.25} & 0.05 & 6 & $3 m$ 32s & 434 & 8 & $15 \mathrm{~m} \mathrm{49s}$ & 916 \\
\hline & 0.10 & 6 & $3 \mathrm{~m} \mathrm{22s}$ & 434 & 8 & $12 \mathrm{~m} \mathrm{14s}$ & 916 \\
\hline & 0.20 & 6 & $3 \mathrm{~m} \mathrm{22s}$ & 434 & 8 & $10 \mathrm{~m} 41 \mathrm{~s}$ & 916 \\
\hline
\end{tabular}




\section{Stabilized Method}

\begin{tabular}{|c|c|c|c|c|c|c|c|}
\hline \multirow[b]{2}{*}{$\epsilon$} & \multirow[b]{2}{*}{$\Delta$} & \multicolumn{3}{|c|}{ Uncongested } & \multicolumn{3}{|c|}{ Congested } \\
\hline & & iter. & time & pool size & iter. & time & pool size \\
\hline \multirow{3}{*}{1.00} & & 15 & $19 \mathrm{~m} \mathrm{15s}$ & 357 & 35 & $1 \mathrm{~h} 7 \mathrm{~m} 12 \mathrm{~s}$ & 560 \\
\hline & & 14 & $12 \mathrm{~m} 31 \mathrm{~s}$ & 390 & 37 & $50 \mathrm{~m} 17 \mathrm{~s}$ & 565 \\
\hline & 0.20 & 11 & $10 m 59 s$ & 397 & 26 & $44 m 53 s$ & 624 \\
\hline \multirow{3}{*}{0.75} & 0.05 & 10 & $8 m$ 43s & 403 & 21 & $45 \mathrm{~m} 41 \mathrm{~s}$ & 642 \\
\hline & & 12 & $8 m 38 s$ & 400 & 21 & $40 \mathrm{~m} 11 \mathrm{~s}$ & 642 \\
\hline & & 9 & $7 \mathrm{~m} \mathrm{37s}$ & 407 & 21 & $39 m$ 8s & 642 \\
\hline \multirow{3}{*}{0.50} & 0.05 & 7 & $4 m 27 s$ & 397 & 11 & $23 m$ 15s & 724 \\
\hline & & 7 & $3 m 58 s$ & 397 & 11 & $21 \mathrm{~m} \mathrm{2s}$ & 724 \\
\hline & & 7 & $3 \mathrm{~m} \mathrm{51s}$ & 397 & 11 & $19 \mathrm{~m} \mathrm{29s}$ & 724 \\
\hline \multirow{3}{*}{0.25} & 0.05 & 6 & $3 m$ 32s & 434 & 8 & $15 \mathrm{~m} \mathrm{49s}$ & 916 \\
\hline & & 6 & $3 m 22 s$ & 434 & 8 & $12 \mathrm{~m} 14 \mathrm{~s}$ & 916 \\
\hline & 0.20 & 6 & $3 m 22 s$ & 434 & 8 & $10 \mathrm{~m} \mathrm{41s}$ & 916 \\
\hline
\end{tabular}




\section{Methods Comparison}

\begin{tabular}{ccccccccc}
\hline & \multicolumn{3}{c}{ Uncongested } & & \multicolumn{3}{c}{ Congested } \\
\cline { 2 - 3 } \cline { 6 - 8 } & iter. & time & pool size & & iter. & time & pool size \\
\hline without & 2 & $9 \mathrm{~s}$ & 122 & & 3 & $13 \mathrm{~s}$ & 132 \\
ARMP & 2 & $9 \mathrm{~s}$ & 122 & & 4 & $17 \mathrm{~s}$ & 226 \\
ARMP+SRMP1 & 8 & $42 \mathrm{~s}$ & 85 & & 10 & $59 \mathrm{~s}$ & 117 \\
ARMP+SRMP2 & 3 & $14 \mathrm{~s}$ & 79 & & 3 & $16 \mathrm{~s}$ & 159 \\
\hline
\end{tabular}

\begin{tabular}{|c|c|c|c|c|c|c|}
\hline & \multicolumn{3}{|c|}{ Uncongested } & \multicolumn{3}{|c|}{ Congested } \\
\hline & iter. & time & pool size & iter. & time & pool size \\
\hline without & 4 & $2 \mathrm{~m} \mathrm{18s}$ & 587 & 7 & $6 \mathrm{~m} \mathrm{18s}$ & 1300 \\
\hline ARMP & 5 & $2 \mathrm{~m} \mathrm{29s}$ & 608 & 9 & $6 \mathrm{~m} \mathrm{51s}$ & 1546 \\
\hline ARMP+SRMP1 & 13 & $9 \mathrm{~m} \mathrm{56s}$ & 429 & 26 & $28 \mathrm{~m} \mathrm{51s}$ & 1107 \\
\hline ARMP+SRMP2 & 5 & $2 \mathrm{~m} \mathrm{59s}$ & 450 & 11 & $8 \mathrm{~m} 3 \mathrm{~s}$ & 968 \\
\hline
\end{tabular}




\section{Methods Comparison}

\begin{tabular}{ccccccccc}
\hline & \multicolumn{3}{c}{ Uncongested } & & \multicolumn{3}{c}{ Congested } \\
\cline { 2 - 3 } \cline { 6 - 8 } & iter. & time & pool size & & iter. & time & pool size \\
\hline without & 2 & $9 \mathrm{~s}$ & 122 & & 3 & $13 \mathrm{~s}$ & 132 \\
ARMP & 2 & $9 \mathrm{~s}$ & 122 & & 4 & $17 \mathrm{~s}$ & 226 \\
ARMPPSRMP1 & 8 & $42 \mathrm{~s}$ & 85 & & 10 & $59 \mathrm{~s}$ & 117 \\
ARMPTSRMP2 & 3 & $14 \mathrm{~s}$ & 79 & & 3 & $16 \mathrm{~s}$ & 159 \\
\hline
\end{tabular}

\begin{tabular}{|c|c|c|c|c|c|c|}
\hline & \multicolumn{3}{|c|}{ Uncongested } & \multicolumn{3}{|c|}{ Congested } \\
\hline & iter. & time & pool size & iter. & time & pool size \\
\hline without & 4 & $2 \mathrm{~m} \mathrm{18s}$ & 587 & 7 & $6 \mathrm{~m} \mathrm{18s}$ & 1300 \\
\hline ARMP & 5 & $2 \mathrm{~m} \mathrm{29s}$ & 608 & 9 & $6 \mathrm{~m} \mathrm{51s}$ & 1546 \\
\hline & 13 & $9 m 56 s$ & 429 & 26 & $28 \mathrm{~m} \mathrm{51s}$ & 1107 \\
\hline & 5 & $2 \mathrm{~m} \mathrm{59s}$ & 450 & 11 & $8 \mathrm{~m} 3 \mathrm{~s}$ & 968 \\
\hline
\end{tabular}




\section{Aggregated Method}

\begin{tabular}{|c|c|c|c|c|c|c|c|c|c|c|}
\hline \multicolumn{3}{|c|}{ Instance } & \multicolumn{2}{|c|}{ Column Generation } & \multirow[b]{2}{*}{$\Omega_{1}$} & \multirow[b]{2}{*}{$\mathrm{lb}$} & \multirow[b]{2}{*}{$u b$} & \multirow[b]{2}{*}{ gap1 } & \multirow[b]{2}{*}{ gap2 } & \multirow[b]{2}{*}{ gap } \\
\hline \# vessels & \# sections & congestion & time & \# iter. & & & & & & \\
\hline \multirow{4}{*}{10} & \multirow{2}{*}{10} & no & $2 \mathrm{~m} \mathrm{36s}$ & 5 & 608 & 197 & 206 & $2 \%$ & $2 \%$ & $4 \%$ \\
\hline & & yes & $7 \mathrm{~m} \mathrm{53s}$ & 9 & 1546 & 213 & 221 & $3 \%$ & $1 \%$ & $4 \%$ \\
\hline & \multirow{2}{*}{20} & no & $5 \mathrm{~m} \mathrm{10s}$ & 4 & 726 & 306 & 310 & $0 \%$ & $1 \%$ & $1 \%$ \\
\hline & & yes & $22 \mathrm{~m} \mathrm{09s}$ & 14 & 2054 & 311 & 316 & $2 \%$ & $0 \%$ & $2 \%$ \\
\hline \multirow{4}{*}{15} & \multirow{2}{*}{10} & no & $3 \mathrm{~m} \mathrm{23s}$ & 5 & 1106 & 293 & 304 & $3 \%$ & $1 \%$ & $4 \%$ \\
\hline & & yes & $22 \mathrm{~m} \mathrm{02s}$ & 7 & 2482 & 314 & 338 & $4 \%$ & $3 \%$ & $7 \%$ \\
\hline & \multirow{2}{*}{20} & no & $15 \mathrm{~m} \mathrm{06s}$ & 6 & 1533 & 447 & 455 & $1 \%$ & $1 \%$ & $2 \%$ \\
\hline & & yes & $1 \mathrm{~h} 5 \mathrm{~m} 54 \mathrm{~s}$ & 15 & 3362 & 457 & 473 & $2 \%$ & $1 \%$ & $3 \%$ \\
\hline \multirow{4}{*}{20} & \multirow{2}{*}{10} & no & $3 \mathrm{~m} 58 \mathrm{~s}$ & 7 & 1330 & 372 & 389 & $3 \%$ & $1 \%$ & $4 \%$ \\
\hline & & yes & $34 \mathrm{~m} \mathrm{24s}$ & 8 & 3847 & 396 & 425 & $6 \%$ & $1 \%$ & $7 \%$ \\
\hline & \multirow{2}{*}{20} & no & $14 \mathrm{~m} \mathrm{24s}$ & 6 & 1921 & 562 & 568 & $1 \%$ & $0 \%$ & $1 \%$ \\
\hline & & yes & 4h $00 \mathrm{~m} \mathrm{00s}$ & 17 & 5406 & 583 & 622 & - & - & $6 \%$ \\
\hline
\end{tabular}




\section{Aggregated Method}

\begin{tabular}{|c|c|c|c|c|c|c|c|c|c|c|}
\hline \multicolumn{3}{|c|}{ Instance } & \multicolumn{2}{|c|}{ Column Generation } & \multirow[b]{2}{*}{$\Omega_{1}$} & \multirow[b]{2}{*}{$\mathrm{lb}$} & \multirow[b]{2}{*}{$u b$} & \multirow[b]{2}{*}{ gap1 } & \multirow[b]{2}{*}{ gap2 } & \multirow[b]{2}{*}{ gap } \\
\hline \# vessels & \# sections & congestion & time & \# iter. & & & & & & \\
\hline & & & $2 \mathrm{~m} \mathrm{36s}$ & 5 & 608 & 197 & 206 & $2 \%$ & $2 \%$ & $4 \%$ \\
\hline & & & $7 \mathrm{~m} \mathrm{53s}$ & 9 & 1546 & 213 & 221 & $3 \%$ & $1 \%$ & $4 \%$ \\
\hline & & & $5 \mathrm{~m} \mathrm{10s}$ & 4 & 726 & 306 & 310 & $0 \%$ & $1 \%$ & $1 \%$ \\
\hline & & & $22 \mathrm{~m} \mathrm{09s}$ & 14 & 2054 & 311 & 316 & $2 \%$ & $0 \%$ & $2 \%$ \\
\hline \multirow{4}{*}{15} & \multirow{2}{*}{10} & no & $3 \mathrm{~m} \mathrm{23s}$ & 5 & 1106 & 293 & 304 & $3 \%$ & $1 \%$ & $4 \%$ \\
\hline & & & $22 \mathrm{~m} \mathrm{02s}$ & 7 & 2482 & 314 & 338 & $4 \%$ & $3 \%$ & $7 \%$ \\
\hline & & & $15 \mathrm{~m} \mathrm{06s}$ & 6 & 1533 & 447 & 455 & $1 \%$ & $1 \%$ & $2 \%$ \\
\hline & & & $1 \mathrm{~h} 5 \mathrm{~m} \mathrm{54s}$ & 15 & 3362 & 457 & 473 & $2 \%$ & $1 \%$ & $3 \%$ \\
\hline \multirow{4}{*}{20} & \multirow{2}{*}{10} & no & $3 \mathrm{~m} \mathrm{58s}$ & 7 & 1330 & 372 & 389 & $3 \%$ & $1 \%$ & $4 \%$ \\
\hline & & yes & $34 \mathrm{~m} \mathrm{24s}$ & 8 & 3847 & 396 & 425 & $6 \%$ & $1 \%$ & $7 \%$ \\
\hline & \multirow{2}{*}{20} & no & $14 \mathrm{~m} \mathrm{24s}$ & 6 & 1921 & 562 & 568 & $1 \%$ & $0 \%$ & $1 \%$ \\
\hline & & yes & $4 \mathrm{~h} 00 \mathrm{~m} 00 \mathrm{~s}$ & 17 & 5406 & 583 & 622 & - & - & $6 \%$ \\
\hline
\end{tabular}




\section{Branch and Bound}

\begin{tabular}{|c|c|c|c|c|c|c|c|}
\hline \multicolumn{3}{|c|}{ Instance } & \multicolumn{5}{|c|}{ Branch \& Bound } \\
\hline \# vessels & \# sections & congestion & time & value & \# of nodes & \# of $\mu$ & $\#$ of $\lambda$ \\
\hline \multirow{4}{*}{10} & \multirow{2}{*}{10} & no & $1 \mathrm{~m} \mathrm{03s}$ & 202 & 13 & 6 & 0 \\
\hline & & yes & $3 \mathrm{~m} \mathrm{35s}$ & 219 & 15 & 7 & 0 \\
\hline & \multirow{2}{*}{20} & no & $9 m$ 35s & 306 & 3 & 1 & 0 \\
\hline & & yes & - & - & - & - & - \\
\hline \multirow{4}{*}{15} & \multirow{2}{*}{10} & no & $2 \mathrm{~m} \mathrm{27s}$ & 301 & 35 & 17 & 0 \\
\hline & & yes & - & - & - & - & - \\
\hline & \multirow{2}{*}{20} & no & $27 \mathrm{~m} \mathrm{13s}$ & 450 & 15 & 7 & 0 \\
\hline & & yes & - & - & - & - & - \\
\hline \multirow{4}{*}{20} & \multirow{2}{*}{10} & no & - & - & - & - & - \\
\hline & & yes & - & - & - & - & - \\
\hline & \multirow{2}{*}{20} & no & - & - & - & - & - \\
\hline & & yes & - & - & - & - & - \\
\hline
\end{tabular}




\section{Initial vs. Optimal}

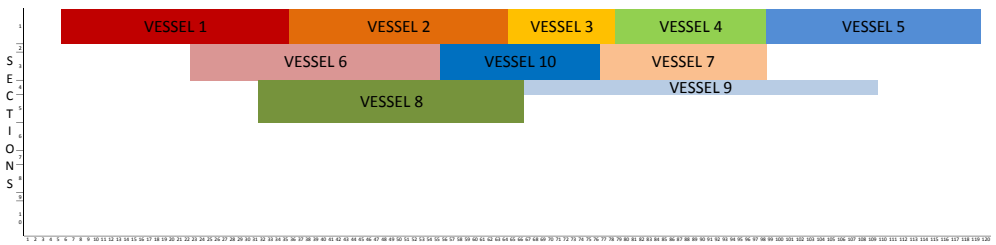

TIME HORIZON

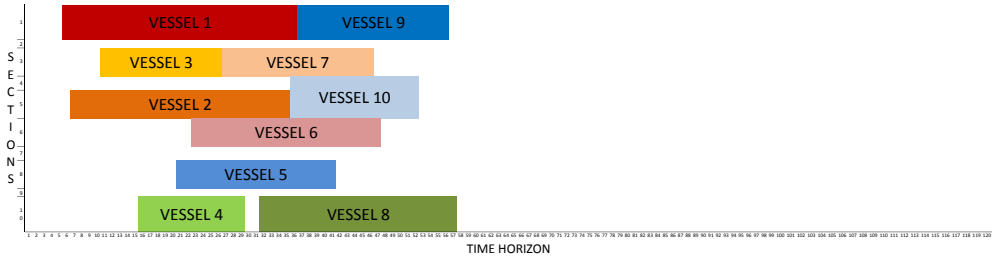

TRANSP-DR

(PPFl

ECOLE POLYTICHNIQUE FEDTRLI DE LAUSANNE

$30 / 35$ 


\section{Problem Definition}

3 Branch \& Price

4. Results

5 Conclusion

6 Future Work

$S_{\text {TRANSP-OR }}$ 


\section{Conclusion}

- proven re-formulation

- "fast" master problem

- "poor" sub-problem

- good (small number of nodes) and bad (time) performance of Branch and Bound

- fast convergence of Column Generation (number of iterations)

- tight bounds (upper and lower) of Column Generation

- dual variables not oscilating

- "hidden" power of aggregated method (although failed for $\Omega$ ) 
4. Results

5 Conclusion

6 Future Work

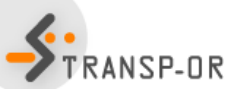




\section{Future Work}

\section{Model:}

- simultaneous loading and unloading

- several cargo on the vessel

- uncertainity, disruptions

- (continous quay)

\section{Branch and Price:}

- better heuristic for initial solution

- heuristic/dynamic programming to solve sub-problem

- heuristic to find integral (sub-optimal) solution or Branch and Cut to find optimal solution, instead of Branch and Bound 


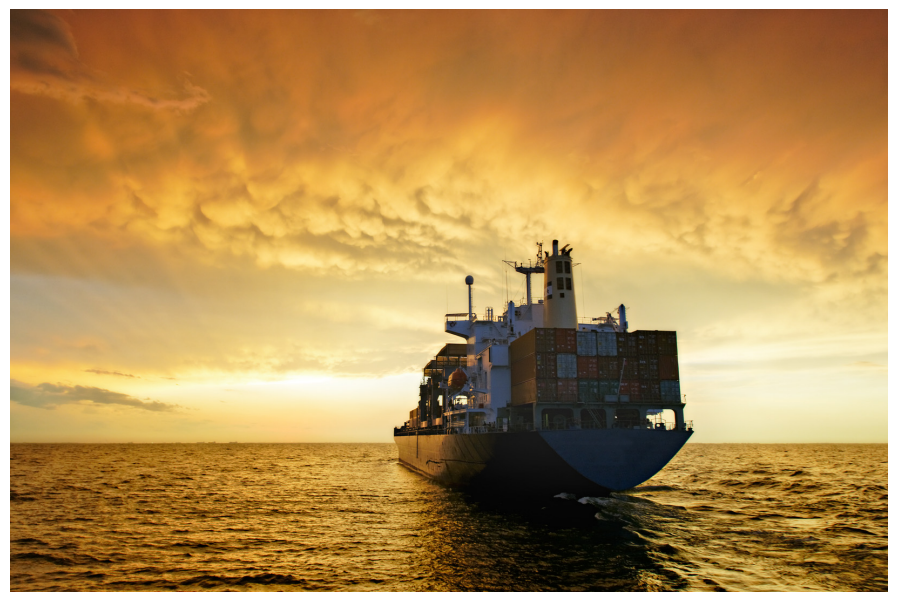

Thank you for your attention. 


\section{Model Groback I}

$$
\begin{array}{rc}
\min \sum_{i}\left(m_{i}-A_{i}+c_{i}\right) & \\
\text { s.t. } m_{i}-A_{i} \geq 0 & \forall i \in N \\
\sum_{k \in M}\left(s_{k}^{j} b_{k}\right)+B\left(1-y_{i j}\right) \geq \sum_{k \in M}\left(s_{k}^{i} b_{k}\right)+L_{i} & \forall i, j \in N, i \neq j \\
m_{j}+B\left(1-z_{i j}\right) \geq m_{i}+c_{i} & \forall i \in N, \forall j \in N, i \neq j \\
y_{i j}+y_{j i}+z_{i j}+z_{j i} \geq 1 & \forall i \in N, \forall j \in N, i \neq j \\
\sum_{k \in M} s_{k}^{i}=1 & \forall i \in N \\
\sum_{k \in M}\left(s_{k}^{i} b_{k}\right)+L_{i} \leq L &
\end{array}
$$




\section{Model đGo back II}

$$
\begin{array}{cc}
\sum_{p \in M}\left(\delta_{i \ell k} s_{\ell}^{i}\right)=x_{i k} & \forall i \in N, \forall k \in M \\
\left(d_{k}-D_{i}\right) x_{i k} \geq 0 & \forall i \in N, \forall k \in M \\
c_{i} \geq h_{i k}^{w} \rho_{i \ell k} Q_{i}-B\left(1-s_{\ell}^{i}\right) & \forall i \in N, \forall I \in M, \\
h_{i k}^{w}=\alpha_{i k}^{w}+\beta_{i k}^{w} & \forall k \in M, \forall w \in W_{i} \\
\beta_{i k}^{w}=V_{w} r_{k}^{i} & \forall w \in W_{i}, \forall k \in M \\
r_{k}^{i}=\sum_{p \in P}\left(r_{k}^{p} \lambda_{i p}\right) / Q_{i} & \forall i \in N, \forall k \in M \\
\sum_{p \in p} \phi_{i p} \leq F & \forall i \in W_{i}, \forall k \in M(13)
\end{array}
$$




\section{Model बGo back III}

$$
\begin{array}{cc}
\pi_{w}^{p}+\pi_{u}^{q} \leq 1 & \forall w \in W, \forall u \in \bar{W}(w), \\
\sum_{i \in N} \omega_{t}^{i p} \leq 1 & \forall p \in P, \forall q \in \bar{P}(p) \\
\sum_{w \in W} \pi_{w}^{p} \leq 1 & \forall p \in P, \forall t \in H \\
\phi_{i p} \leq \pi_{w}^{p} & \forall i \in N, \forall w \in W_{i}, \forall p \in P(17) \\
\omega_{t}^{i p} \geq \phi_{i p}+\theta_{i t}-1 & \forall i \in N, \forall p \in P, \forall t \in H(20) \\
\omega_{t}^{i p} \leq \phi_{i p} & \forall i \in N, \forall p \in P, \forall t \in H(21) \\
\omega_{t}^{i p} \leq \theta_{i t} & \forall i \in N, \forall p \in P, \forall t \in H \text { (22) } \\
\sum_{t \in H} \theta_{i t}=c_{i} & \forall i \in N
\end{array}
$$




\section{Model crobad IV}

$$
\begin{array}{rc}
t+B\left(1-\theta_{i t}\right) \geq m_{i}+1 & \forall i \in N, \forall t \in H \\
t \leq m_{i}+c_{i}++B\left(1-\theta_{i t}\right) & \forall i \in N, \forall t \in H \\
Q_{i}=\sum_{p \in P} \lambda_{i p} & \forall i \in N \\
\lambda_{i p} \leq \phi_{i p} Q_{i} & \forall i \in N, \forall p \in P \\
\phi_{i p} \leq \lambda_{i p} & \forall p \in P \\
\lambda_{i p} \leq \sum_{w \in W_{i}} \sum_{t \in H}\left(R_{w} \omega_{t}^{i p}+B\left(1-\pi_{w}^{p}\right)\right) & \forall i \in N, \forall p \in P \\
s_{k}^{i} \in\{0,1\} & \forall i \in N, \forall k \in M \\
x_{i k} \in\{0,1\} & \forall i \in N, \forall k \in M \\
y_{i j} \in\{0,1\} & \forall i, j \in N
\end{array}
$$




\section{Model (Go back V}

$$
\begin{aligned}
& z_{i j} \in\{0,1\} \\
& \pi_{w}^{p} \in\{0,1\} \\
& \omega_{t}^{i p} \in\{0,1\} \\
& \phi_{i p} \in\{0,1\} \\
& \theta_{i t} \in\{0,1\}
\end{aligned}
$$

$$
\begin{gathered}
\forall i, j \in N \\
\forall p \in P, \forall w \in W \\
\forall i \in N, \forall p \in P, \forall t \in H \text { (35) } \\
\forall i \in N, \forall p \in P \\
\forall i \in N, \forall t \in H
\end{gathered}
$$




\section{Set Partitioning}

$$
\begin{aligned}
\min \sum_{a \in \Omega} c_{a} \cdot \lambda_{a} & \\
\text { s.t. } \sum_{a \in \Omega} A_{a}^{i} \cdot \lambda_{a}=1, & \forall i \in N, \\
\sum_{a \in \Omega} B_{a}^{k t} \cdot \lambda_{a} \leq 1, & \forall k \in K, \forall t \in T, \\
\sum_{a \in \Omega} C_{a}^{l w} \cdot \lambda_{a}-c t_{w} \cdot \mu_{w}^{l} \leq 0, & \forall I \in L, \forall w \in W, \\
\sum_{w \in W} \mu_{w}^{l} \leq 1, & \forall I \in L,
\end{aligned}
$$




\section{Sub-Problem Groback}

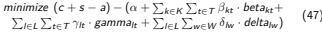

$\begin{aligned} s-a \geq 0, & \\ c \geq h t_{k} \cdot \text { fraction } j k-M \cdot\left(1-s s_{j}\right), & \forall j, k \in K,\end{aligned}$

